\title{
Correlation of primary middle and distal esophageal cancers motion with surrounding tissues using four-dimensional computed tomography
}

This article was published in the following Dove Press journal:

OncoTargets and Therapy

22 June 2016

Number of times this article has been viewed

\author{
Wei Wang' \\ Jianbin $\mathrm{Li}^{1}$ \\ Yingjie Zhang' \\ Qian Shao' \\ Min Xu' \\ Bing Guo' \\ Dongping Shang ${ }^{2}$ \\ 'Department of Radiation Oncology, \\ ${ }^{2}$ Department of Big Bore CT Room, \\ Shandong Cancer Hospital Affiliated \\ to Shandong University, Shandong \\ Academy of Medical Sciences, Jinan, \\ Shandong, People's Republic of China
}

Correspondence: Jianbin Li Department of Radiation Oncology (Chest Section), Shandong Cancer Hospital Affiliated to Shandong University, Shandong Academy of Medical Sciences, Road Jiyan 440, Jinan 250II7, Shandong, People's Republic of China $\mathrm{Tel} / \mathrm{fax}+8653167626130$ Email lijianbin@msn.com
Purpose: To investigate the correlation of gross tumor volume (GTV) motion with the structure of interest (SOI) motion and volume variation for middle and distal esophageal cancers using four-dimensional computed tomography (4DCT).

Patients and methods: Thirty-three patients with middle or distal esophageal carcinoma underwent 4DCT simulation scan during free breathing. All image sets were registered with $0 \%$ phase, and the GTV, apex of diaphragm, lung, and heart were delineated on each phase of the 4DCT data. The position of GTV and SOI was identified in all 4DCT phases, and the volume of lung and heart was also achieved. The phase relationship between the GTV and SOI was estimated through Pearson's correlation test.

Results: The mean peak-to-peak displacement of all primary tumors in the lateral (LR), anteroposterior (AP), and superoinferior (SI) directions was $0.13 \mathrm{~cm}, 0.20 \mathrm{~cm}$, and $0.30 \mathrm{~cm}$, respectively. The SI peak-to-peak motion of the GTV was defined as the greatest magnitude of motion. The displacement of GTV correlated well with heart in three dimensions and significantly associated with bilateral lung in LR and SI directions. A significant correlation was found between the GTV and apex of the diaphragm in SI direction $\left(r_{\text {left }}=0.918\right.$ and $\left.r_{\text {right }}=0.928\right)$. A significant inverse correlation was found between GTV motion and varying lung volume, but the correlation was not significant with heart $\left(r_{\mathrm{LR}}=-0.530, r_{\mathrm{AP}}=-0.531\right.$, and $\left.r_{\mathrm{SI}}=-0.588\right)$ during respiratory cycle.

Conclusion: For middle and distal esophageal cancers, GTV should expand asymmetric internal margins. The primary tumor motion has quite good correlation with diaphragm, heart, and lung.

Keywords: esophageal neoplasms, 4D-CT, gross tumor volume, displacement correlation

\section{Introduction}

As one of the major health hazards in the People's Republic of China, esophageal cancer at locally advanced or advanced stage occurs in the vast majority of patients. ${ }^{1}$ Radiotherapy combined with a systemic approach plays an important role in the treatment management of inoperable disease. ${ }^{2,3}$ Concurrent chemoradiotherapy has been associated with improvement in local and regional control. ${ }^{4,5}$ To improve local control in radiotherapy, several methods have been developed to increase the tumor dose and reduce the dose to normal tissue, such as intensity-modulated radiotherapy, respiratory gating radiotherapy, image-guided radiotherapy, and real-time tumor tracking radiotherapy. ${ }^{6-8}$ The intrafractional tumor motion caused by respiration, cardiac impulse, or peristaltic movement of the digestive tract exists, when the esophageal cancer patient is being irradiated, ${ }^{9-11}$ especially for middle and distal esophageal tumors. 
To examine the motion of esophageal tumors during respiratory period, Yaremko et al ${ }^{12}$ used a four-dimensional computed tomography (4DCT) that demonstrated an average movement of $0.13 \pm 0.006 \mathrm{~cm}$ in the $x$-direction, $0.23 \pm 0.01 \mathrm{~cm}$ in the $y$-direction, and $0.71 \pm 0.02 \mathrm{~cm}$ in the $z$-direction for distal esophagus cancers.

Under the assumption that the three-dimensional computed tomography (3DCT) images represent the average position of the tumor, the radiation target and the treatment planning are typically performed on the basis of structures identified in a computed tomography (CT) scan obtained while the patient breathes freely. However, 3DCT images display the tumor during a certain moment of the breathing cycle, ie, randomly in any single respiratory phase or midway between two phases during free breathing. Respirationsynchronized 4DCT images were generated from the 3DCT images providing the three spatial dimensions and the respiratory signal providing the fourth temporal dimension. ${ }^{13,14}$ Therefore, 4DCT has enabled more accurate measurement of the tumor movement and volume variance, potentially to determine patient-specific internal target volume (ITV) and to correlate the magnitude of respiration-induced tumor motion and volume variation with the structures nearby.

Breathing control and respiratory gating can decrease the effect of tumor mobility in radiotherapy for thoracic and abdominal tumors, ${ }^{15-18}$ but these techniques in radiotherapy for middle and distal esophageal cancers were performed at relatively few institutions. The feasibility depends on the strong correlation between the intrafractional tumor motion and respiration variability during the treatment course. In this study, tumor centroid motion was acquired during the whole breathing cycle, and the magnitude of tumor motion was correlated with the movement magnitude of the structure of interest (SOI), including the apex of diaphragm, lung, and heart. On the basis of this correlation, we propose to determine and ascertain the intrafraction tumor motion due to breathing and heartbeat.

\section{Patients and methods}

\section{Patient characteristics}

Thirty-three patients with a diagnosis of esophageal carcinoma located in the middle or distal thoracic esophagus underwent 4DCT simulation scanning for radiation treatment planning. Twenty-three patients had primary tumors in the middle third of the esophagus, and ten patients had primary tumors in the distal third of the esophagus. Adenocarcinoma in eight patients and squamous cell carcinoma in 25 patients were pathologically confirmed. Twenty-eight men and five women with a median age of 65 years (range, 49-82 years) were included. Patients with poor pulmonary function were excluded. Written informed consent was obtained from all the patients before undergoing further imaging during radiotherapy on an institutional protocol approved by the Shandong Cancer Hospital and Institute Ethics Committee.

\section{DCT data acquisition}

The patients were immobilized in a vacuum bag in the supine position with their arms above their head. 4DCT images were acquired for each patient under free breathing using a 16-slice CT scanner (Philips Medical System, Cleveland, OH, USA). Simultaneously, the respiratory signal was acquired via the real-time position management (RPM) system (Varian Medical Systems, Palo Alto, CA, USA), by tracking the displacement of the infrared markers placed on the epigastric region of the patient. The CT images and the RPM file containing the patient respiratory signal were uploaded into the GE Advantage Workstation (GE Advantage 4D software; GE Healthcare Bio-Sciences Corp., Piscataway, NJ, USA). Then, the reconstructed 4DCT images were sorted into ten respiratory phases labeled as $0 \%-90 \%$ on the basis of triggered signal, and $0 \%$ and $50 \%$ correspond to the end inspiration and end expiration, respectively. The 4DCT images were reconstructed using a thickness of $3 \mathrm{~mm}$ and then exported to the Eclipse treatment planning system (Eclipse 8.6; Varian Medical Systems).

\section{Delineation of the gross tumor volume and SOI}

To eliminate interobserver variations, the same radiation oncologist delineated the gross tumor volume (GTV), heart, lung, and apex of the diaphragm. Each volume was contoured using the mediastinal window/level. The corresponding fullinspiration centroid position and volume were acquired by Varian's Eclipse 8.6 treatment planning system (Figure 1), and the displacement of each GTV and SOI in the lateral (LR), anteroposterior (AP), and superoinferior (SI) directions was calculated directly. The GTV only included the primary gross esophageal tumor. The cranial portion of GTV was determined as either the most proximal portion with the thickness $>0.5 \mathrm{~cm}$ or following a region of dilation. The following esophagus was considered GTV until the wall returned to $0.3 \mathrm{~cm}$ thickness or the gastroesophageal junction was reached. ${ }^{12}$

\section{Statistical methods}

Statistical analysis was computed with the SPSS 19.0 software (IBM Corporation, Armonk, NY, USA). Paired sample $t$-test was performed to determine statistical significance 

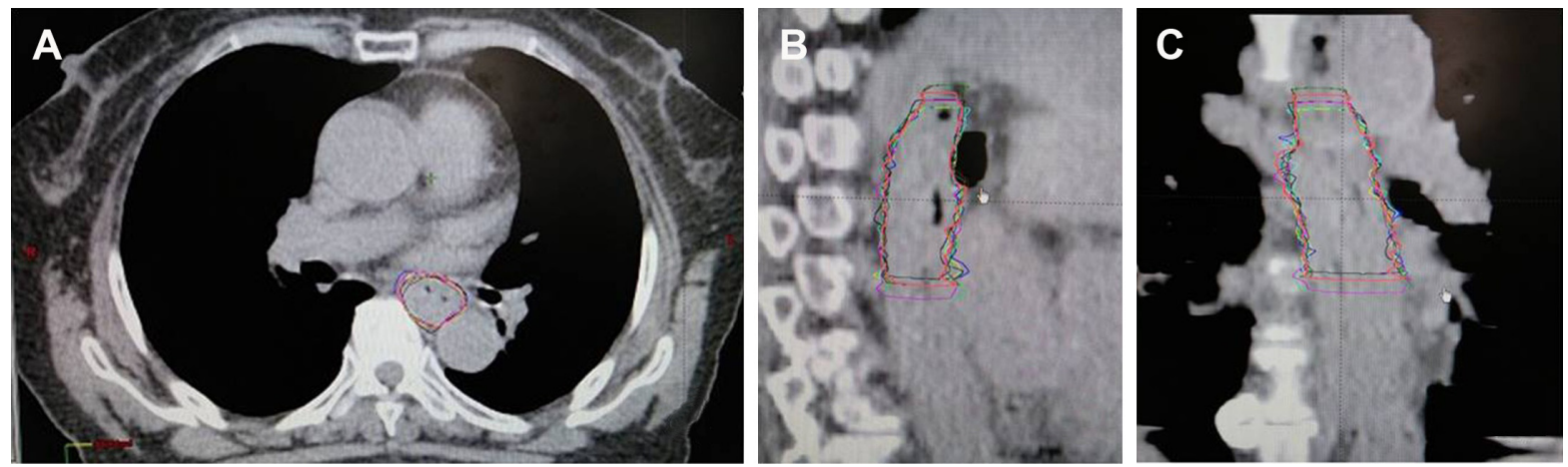

Figure I The GTV was delineated on each phase of the 4DCT data (all image sets were registered with El phase). Note: A, B, and $\mathbf{C}$ are the respective axial, sagittal, and coronal view of the patient exhibiting the GTV in ten phases. Abbreviations: 4DCT, four-dimensional computed tomography; El, end inspiration; GTV, gross tumor volume.

in the difference of the three-dimensional displacement. Pearson's correlation test was used to study the relationship between the motion of GTV and SOI during free breathing, and the relationship between the GTV motion and SOI volume variance to evaluate whether the three-dimensional motion and volume variance of SOI synchronized with the corresponding GTV motion. Differences were considered significant for $P<0.05$. All statistical analyses were performed using the SPSS 17.0 software package.

\section{Results GTV motion on free-breathing 4DCT} scans

The mean peak-to-peak motion of the primary tumor in the LR, AP, and SI directions of the 33 patients was $0.13 \pm 0.02 \mathrm{~cm}, 0.20 \pm 0.04 \mathrm{~cm}$, and $0.30 \pm 0.05 \mathrm{~cm}$, respectively. The SI peak-to-peak motion of the GTV was defined as the greatest magnitude of motion $\left(t_{\mathrm{LR}-\mathrm{SI}}=-6.219, p_{\mathrm{LR}-\mathrm{SI}}=0.000\right.$; $\left.t_{\mathrm{AP}-\mathrm{SI}}=-6.682, p_{\mathrm{AP}-\mathrm{SI}}=0.000\right)$.

The $0 \%$ phase of CT images was used for the reference for image registration, and the average amplitude of the tumor motion was assessed in all three directions during the whole breathing cycle. Figures 1 and 2 show the GTV movement in successive phases of free-breathing 4DCT scans. The GTV centroid motion was modeled nearly as a sinusoidal movement with varying asymmetry in three different directions. The variation in the average of extreme tumor positions was comparable with the corresponding values of the actual data of the respiratory motion. For the LR and SI directions, the extreme position of GTV was fitted to the average values, but a little skewing was observed in the AP direction.

\section{Relationship between GTV and SOI positions}

Figure 3 shows that, for both the lung and heart, the centric movement of several millimeters could be observed in the
$\mathrm{LR}, \mathrm{AP}$, and SI directions during respiratory cycle. To assess whether the magnitude of GTV motion correlated with the magnitude of the SOI motion, we calculated Pearson's correlation coefficients and $P$-values in the three orthogonal dimensions (Table 1). The GTV position correlated well with the motion of heart in the three orthogonal dimensions. However, the correlation was not there in the AP direction for the right lung and the left lung. In the LR and SI directions, we have also demonstrated that the GTV movement was attributed to breathing motion. A significant correlation was observed between the GTV and apex of the diaphragm in SI direction ( $r_{\text {left }}=0.958$ and $\left.r_{\text {right }}=0.958\right)$.

\section{Relationship between GTV motion and SOI volume variation}

For the patients undergoing free-breathing 4DCT scans, the GTV motion in three-dimensional directions all correlated well with the change in total lung volume throughout the respiratory cycle (Table 2). Table 2 also shows the correlation of the GTV motion and heart volume variation during

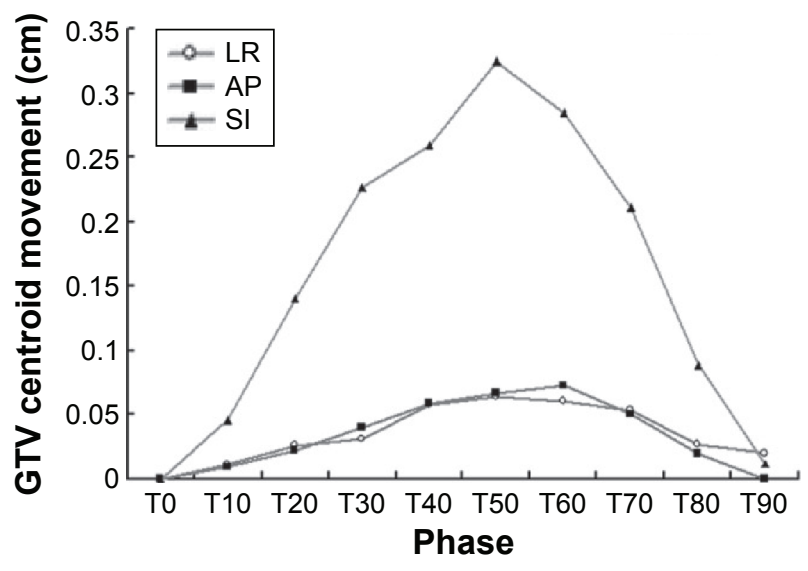

Figure 2 GTV movement in successive phases of free breathing 4DCT scans. Abbreviations: AP, anteroposterior; 4DCT, four-dimensional computed tomography; GTV, gross tumor volume; LR, lateral; SI, superoinferior. 


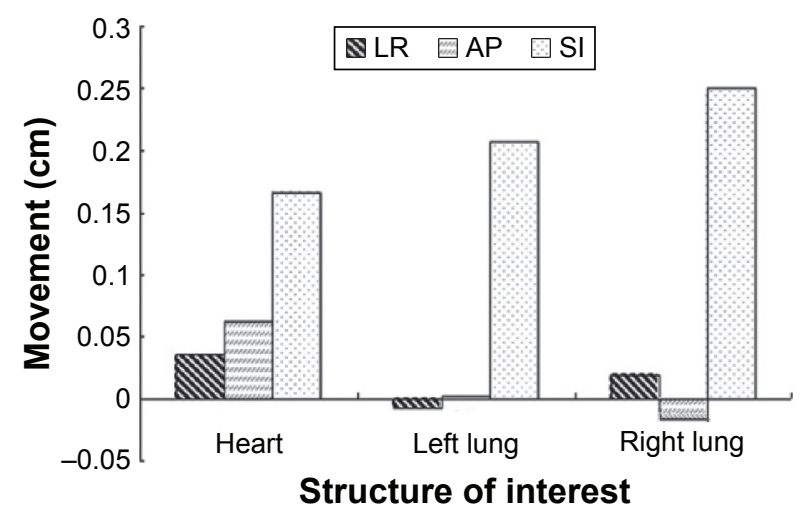

Figure 3 The mean peak-to-peak motion of the lung and heart in the three directions.

Abbreviations: AP, anteroposterior; LR, lateral; SI, superoinferior.

the normal respiratory cycle, and no meaningful correlation could be established with them.

\section{Discussion}

Esophageal intrafraction motion during irradiation delivery is one of the geometrical uncertainties that may affect the therapeutic effects. We evaluated the middle and distal esophageal tumors' motion using 4DCT and showed the centroid movements of several millimeters in the LR, AP, and SI directions for both GTV and SOI. During free breathing, the respiration-induced tumor motion characteristic in three spatial dimensions was not entirely consistent (Figure 2), and the GTV had the largest movement in the SI direction (average $0.30 \mathrm{~cm}$ ). Our results were similar with others,,$^{10,12,19,20}$ for example, Patel et al ${ }^{19}$ measured the motion of primary tumors located in the proximal, middle, or distal thoracic esophagus and found that the peak-to-peak displacement of all primary tumors in the SI, AP, and LR dimensions was $0.80 \pm 0.45 \mathrm{~cm}$, $0.28 \pm 0.20 \mathrm{~cm}$, and $0.22 \pm 0.23 \mathrm{~cm}$, respectively. Although our results were somewhat smaller, the ranking order remains the same (SI > LR and AP). Therefore, asymmetric margins are recommended because of the variations in tumor motion. The major discrepancies might associate with the race difference and location of the tumor. The main constituent of

Table I Pearson's correlation coefficients between GTV motion and SOI motion in the three orthogonal dimensions

\begin{tabular}{|c|c|c|c|c|c|c|}
\hline \multirow[t]{2}{*}{ sol } & \multicolumn{2}{|l|}{ LR } & \multicolumn{2}{|l|}{ AP } & \multicolumn{2}{|l|}{ SI } \\
\hline & $r$ & $P$-value & $r$ & $P$-value & $r$ & $P$-value \\
\hline Left lung & -0.889 & 0.001 & 0.081 & 0.824 & 0.996 & 0.000 \\
\hline Right lung & 0.945 & 0.000 & -0.607 & 0.063 & 0.995 & 0.000 \\
\hline Heart & 0.821 & 0.004 & 0.946 & 0.000 & 0.953 & 0.000 \\
\hline
\end{tabular}

Abbreviations: AP, anteroposterior; GTV, gross tumor volume; LR, lateral; $\mathrm{SI}$, superoinferior; SOI, structure of interest.
Table 2 Pearson's correlation coefficients between GTV motion in the three orthogonal dimensions and SOI volume variation

\begin{tabular}{|c|c|c|c|c|c|c|}
\hline \multirow[t]{2}{*}{ SOI } & \multicolumn{2}{|l|}{ LR } & \multicolumn{2}{|l|}{ AP } & \multicolumn{2}{|l|}{ SI } \\
\hline & $r$ & $P$-value & $r$ & $P$-value & $r$ & $P$-value \\
\hline Left lung & -0.957 & 0.000 & -0.983 & 0.000 & -0.982 & 0.000 \\
\hline Right lung & -0.938 & 0.000 & -0.981 & 0.000 & -0.992 & 0.000 \\
\hline Heart & -0.530 & 0.115 & -0.531 & 0.114 & -0.588 & 0.074 \\
\hline
\end{tabular}

Abbreviations: AP, anteroposterior; GTV, gross tumor volume; LR, lateral; $\mathrm{SI}$, superoinferior; SOI, structure of interest.

our patients' primary tumors was located in the middle third of the esophagus, whereas Patel et a ${ }^{19}$ reported that $83.33 \%$ of patients had primary tumors in the distal third of the esophagus. We quantified the mean distal esophageal cancer motion of $0.26 \mathrm{~cm}$ in the LR direction, $0.29 \mathrm{~cm}$ in the AP direction, and $0.53 \mathrm{~cm}$ in the SI direction with a maximum of $1.21 \mathrm{~cm}$.

4DCT images, which involve up to ten times more data than traditional 3DCT scan, can be used to visualize breathing-induced tumor motion and deformation of the breathing cycle, making it possible to reconstruct patient-specific target volume for precise radiotherapy. ${ }^{21-23}$ Our findings revealed that during free breathing, the tumor motion in the LR and SI directions was fitted to the actual data of the sinusoidal model of respiratory motion. However, it was also noted that in the AP direction, the phase relationship between GTV motion and respiration was not always the same, but the extreme phase was close to the $50 \%$ phase. It may possibly be caused by the hysteresis effects during CT scanning, the same as observed in lung tumors. ${ }^{8,24} \mathrm{~A}$ recent study by Yamashita et al ${ }^{11}$ had corroborated that motion in the cranialcaudal direction showed a strong correlation $\left(R^{2}>0.4\right)$ with the respiratory curve in most markers ( $73 \%$ ), and only $23 \%$ and $50 \%$ for the LR and AP directions, respectively, investigated by the 3D movement of internal fiducial markers placed near esophageal cancers. On the basis of our findings and the literature, the patient-specific ITV acquired from the two extreme phases could not account for all the intrafraction spatial information on tumor mobility throughout a treatment course.

We assessed the correlation of GTV motion with the displacement of lung and apex of the diaphragm, and the results have shown that the motion of tumor correlated well with both the right lung and the left lung in the LR and SI directions, and in the SI direction, the tumor motion correlated well with apex of the diaphragm. This finding indicated that the RPM system could reliably predict the tumor motion during the CT scan. Hence, for patients with the middle and distal esophageal cancers, breathing control could decrease the respiratory-induced esophageal tumor movement, and these patients were also good candidates for 
respiratory gating. There might be a trend that, for patients with smaller breathing amplitude, ITVs generated from the two extreme 4DCT data phases potentially lead to less geometric miss.

As a tube-shaped and nonrigid organ in the mediastinum, except respiration-induced movement, motion of the heart and aorta resulting from the cardiac cycle, as well as the peristaltic movement of the digestive tract, can lead to motion and volume deformation for the esophagus. ${ }^{9,10}$ In our data, strong correlations were found between the tumor movement and the bilateral lung centroid motion and also showed a good correlation between the GTV motion and the lung volume variation, so even during free breathing, the chest expansion bears the risk for increasing the tumor motion and deformation.

The motion of the tumor located in the middle and distal esophagus correlated well with the heart, which indicated that the tumors located near the heart were affected by the heartbeat. This finding was similar to the lung cancer. ${ }^{8}$ Breathing control, such as active breathing control, has shown a statistically significant reduction of both the chest wall and diaphragm motion. ${ }^{25}$ While the patients hold their breath, their heat rate is also decreased, and the relative and absolute amount of cardiac motion within breath holding stages would be reduced. Breathing control could decrease respiratory-induced esophageal tumor movement. Lorchel et $\mathrm{al}^{18}$ performed a study to confirm the feasibility of breathing control in conformal radiotherapy of esophageal cancer. They found that deep inspiration breath hold and end inspiration breath hold correlated with a $32 \%$ and $20 \%$ decrease in lung $V_{20}$ and a $25 \%$ and $17 \%$ decrease in cardiac $V_{40}$, respectively, as compared to free breathing. Hence, conformal radiotherapy with respiratory gating for esophageal cancer decreases the irradiated dose to organs at risk, and breathing control technique should be used during esophageal cancer irradiation treatment delivery.

Based on the 4DCT simulation scan during free breathing, we measured the GTV and SOI motion. Although the 4DCT simulation may not accurately represent the magnitude of motion occurring during the subsequent daily radiation delivery, respiratory movement is periodic. Consequently, our study provides reasonable correlation of the GTV motion with the interested structure motion and volume variation for the middle and distal esophageal cancers during radiation delivery. A major limitation of our study was that we could not distinguish between the tumor motion caused by respiration and heart motion.

\section{Conclusion}

Tumor motion measured by the normal breathing 4DCT scans was greatest in the SI direction for middle and distal thoracic esophageal cancers. Asymmetric margins for target definition were recommended because of the variation in tumor motion and deformation. Tumor motion due to respiration and heart motion could be relatively accurately detected by 4DCT for the middle and distal esophageal cancers during radiation delivery. So, breathing control or real-time tumor tracking is feasible for the radiotherapy of middle and distal thoracic esophageal cancers. And patient-specific ITVs generated from the two extreme phases of breathing will not account for all intrafraction spatial information.

\section{Acknowledgment}

This study was funded by Medicine and Health Science Technology Development Program of Shandong Province (no 2015WS0160).

\section{Disclosure}

The authors report no conflicts of interest in this work.

\section{References}

1. Esophageal Carcinoma Cooperative Group of Radiation Oncology Society of Chinese Medical Association. Treatment guideline of radiotherapy for Chinese esophageal carcinoma (draft). Chin J Cancer. 2010; 29(10):855-859.

2. Ajani J, Bekaii-Saab T, D'Amico TA, et al. Esophageal cancer clinical practice guidelines. J Natl Compr Canc Netw. 2006;4(4):328-347.

3. Walsh TN, Noonan N, Hollywood D, Kelly A, Keeling N, Hennessy TP. A comparison of multimodal therapy and surgery for esophageal adenocarcinoma. N Engl J Med. 1996;335(7):462-467.

4. Kersting S, Konopke R, Dittert D, et al. Who profits from neoadjuvant radiochemotherapy for locally advanced esophageal carcinoma? J Gastroenterol Hepatol. 2009;24(5):886-895.

5. Sjoquist KM, Burmeister BH, Smithers BM, et al. Survival after neoadjuvant chemotherapy or chemoradiotherapy for resectable oesophageal carcinoma: an updated meta-analysis. Lancet Oncol. 2011 12(7):681-692.

6. Chen YJ, Han C, Liu A, et al. Setup variations in radiotherapy of esophageal cancer: evaluation by daily megavoltage computed tomographic localization. Int J Radiat Oncol Biol Phys. 2007;68(5): 1537-1545.

7. Vosmik M, Petera J, Sirak I, et al. Technological advances in radiotherapy for esophageal cancer. World J Gastroenterol. 2010;16(44): 5555-5564.

8. Seppenwoolde Y, Shirato H, Kitamura K, et al. Precise and real-time measurement of 3D tumor motion in lung due to breathing and heartbeat, measured during radiotherapy. Int J Radiat Oncol Biol Phys. 2002; 53(4):822-834.

9. Hashimoto T, Shirato H, Kato M, et al. Real-time monitoring of a digestive tract marker to reduce adverse effects of moving organs at risk (OAR) in radiotherapy for thoracic and abdominal tumors. Int J Radiat Oncol Biol Phys. 2005;61(5):1559-1564.

10. Zhao KL, Liao Z, Bucci MK, et al. Evaluation of respiratory-induced target motion for esophageal tumors at the gastroesophageal junction. Radiother Oncol. 2007;84(3):283-289. 
11. Yamashita H, Kida S, Sakumi A, et al. Four-dimensional measurement of the displacement of internal fiducial markers during 320-multislice computed tomography scanning of thoracic esophageal cancer. Int J Radiat Oncol Biol Phys. 2011;79(2):588-595.

12. Yaremko BP, Guerrero TM, McAleer MF, et al. Determination of respiratory motion for distal esophagus cancer using four dimensional computed tomography. Int J Radiat Oncol Biol Phys. 2008;70(1): $145-153$.

13. Keall P. 4-dimensional computed tomography imaging and treatment planning. Semin Radiat Oncol. 2004;14(1):81-90.

14. Roland T, Shi C, Liu Y, Crownover R, Mavroidis P, Papanikolaou N. Tradeoffs for assuming rigid target motion in Mlc-based real time target tracking radiotherapy: a dosimetric and radiobiological analysis. Technol Cancer Res Treat. 2010;9(2):199-210.

15. Wong VY, Tung SY, Ng AW, et al. Real-time monitoring and control on deep inspiration breath-hold for lung cancer radiotherapy combination of ABC and external marker tracking. Med Phys. 2010; 37(9):4673-4683.

16. Ford EC, Mageras GS, Yorke E, Rosenzweig KE, Wagman R, Ling CC. Evaluation of respiratory movement during gated radiotherapy using film and electronic portal imaging. Int J Radiat Oncol Biol Phys. 2002; 52(2):522-531.

17. Wagman R, Yorke E, Ford E, et al. Respiratory gating for liver tumors: use in dose escalation. Int J Radiat Oncol Biol Phys. 2003;55(3): 659-668.

18. Lorchel F, Dumas JL, Noël A, Wolf D, Bosset JF, Aletti P. Dosimetric consequences of breath-hold respiration in conformal radiotherapy of esophageal cancer. Phys Med. 2006;22(4):119-126.
19. Patel AA, Wolfgang JA, Niemierko A, Hong TS, Yock T, Choi NC. Implications of respiratory motion as measured by four-dimensional computed tomography for radiation treatment planning of esophageal cancer. Int J Radiat Oncol Biol Phys. 2009;74(1):290-296.

20. Dieleman EM, Senan S, Vincent A, Lagerwaard FJ, Slotman BJ, van Sörnsen de Koste JR. Four-dimensional computed tomographic analysis of esophageal mobility during normal respiration. Int J Radiat Oncol Biol Phys. 2007;67(3):775-780.

21. Gierga DP, Brewer J, Sharp GC, Betke M, Willett CG, Chen GT. The correlation between internal and external markers for abdominal tumors: implications for respiratory gating. Int J Radiat Oncol Biol Phys. 2005;61(5):1551-1558.

22. Admiraal MA, Schuring D, Hurkmans CW. Dose calculations accounting for breathing motion in stereotactic lung radiotherapy based on 4D-CT and the internal target volume. Radiother Oncol. 2008;86(1): 55-60.

23. Roland T, Mavroidis P, Gutierrez A, Goytia V, Papanikolaou N. A radiobiological analysis of the effect of 3D versus 4D image-based planning in lung cancer radiotherapy. Phys Med Biol. 2009;54(18):5509-5523.

24. Mageras GS, Pevsner A, Yorke ED, et al. Measurement of lung tumor motion using respiration-correlated CT. Int J Radiat Oncol Biol Phys. 2004;60(3):933-941.

25. Gagel B, Demirel C, Kientopf A, et al. Active breathing control (ABC): determination and reduction of breathing-induced organ motion in the chest. Int J Radiat Oncol Biol Phys. 2007;67(3):742-749.
OncoTargets and Therapy

\section{Publish your work in this journal}

OncoTargets and Therapy is an international, peer-reviewed, open access journal focusing on the pathological basis of all cancers, potential targets for therapy and treatment protocols employed to improve the management of cancer patients. The journal also focuses on the impact of management programs and new therapeutic agents and protocols on

\section{Dovepress}

patient perspectives such as quality of life, adherence and satisfaction The manuscript management system is completely online and includes a very quick and fair peer-review system, which is all easy to use. Visit http://www.dovepress.com/testimonials.php to read real quotes from published authors. 